\title{
Nonlinear Light Generation at the High Energy Range of a 3D Opal Film
}

\author{
Muriel Botey, ${ }^{1}$ Marc Maymó, ${ }^{2}$ Alberto Molinos-Gómez,${ }^{3}$ Luis A. Dorado, ${ }^{4}$ Ricardo Depine, ${ }^{4}$ \\ Gabriel Lozano, ${ }^{5}$ Agustín Mihi, ${ }^{5}$ Hernán Míguez, ${ }^{5}$ Jordi Martorell,, \\ ${ }^{1}$ Departament de Física i Enginyeria Nuclear, Universitat Politècnica de Catalunya, C/Urgell 187, 08036 Barcelona, Spain. \\ ${ }^{2}$ ICFO-Institut de Ciencies Fotoniques, Parc Mediterrani de la Tecnologia, Av. del Canal Olímpic s/n, 08860 Castelldefels \\ (Barcelona), Spain. \\ ${ }^{3}$ BASF Construction Chemicals Espana, S.L., C/ Basters, 15 E-08184, Palau-solita i Plegamans,Barcelona 08184, Spain \\ ${ }^{4}$ Grupo de Electromagnetismo Aplicado, Departamento de Física, Facultad de Ciencias Exactas y Naturales, Universidad de \\ Buenos Aires, Buenos Aires, Argentina. \\ ${ }^{5}$ Instituto de Ciencia de Materiales de Sevilla, Consejo Superior de Investigaciones Cientificas (CSIC), Américo Vespucio 49, 41092 \\ Sevilla, Spain.
}

Most of the applications based on the special optical properties that photonic crystals (PC) offer to control the interaction between matter and electromagnetic radiation, are based on the periodic structure response at the low energy region of the spectra, where the first order Bragg diffraction takes place. Among those reported applications of PCs some are devoted to the nonlinear (NL) optical processes such as the second harmonic generation ( $\mathrm{SHG})$.

In the present paper we experimentally show that SHG is also possible in a NL artificial opal, at the high energy range, where the system displays a truly 3D behavior. We have fabricated a nonlinear opal film of monodisperse polystyrene spheres coated with a dye nonlinear molecule. We have measured the $\mathrm{SH}$ forward-generated transmitted light upon incidence of a frequency field, propagating close to (111) direction of the crystal. By means of vector KKR method including extinction [1,2], we have determined the phase delay introduced to the specularly reflected and forwardly transmitted electromagnetic fields from our finite photonic structures in that spectral region. We have then calculated the group velocity of the electromagnetic waves from the linear response of the finite crystal which is shown as a function of frequency in Fig.1.a. As seen in Fig.1.b, the observed enhancement of the SHG of light occurs where these phase delays lead to a significant reduction of the group velocity with respect to the average value.
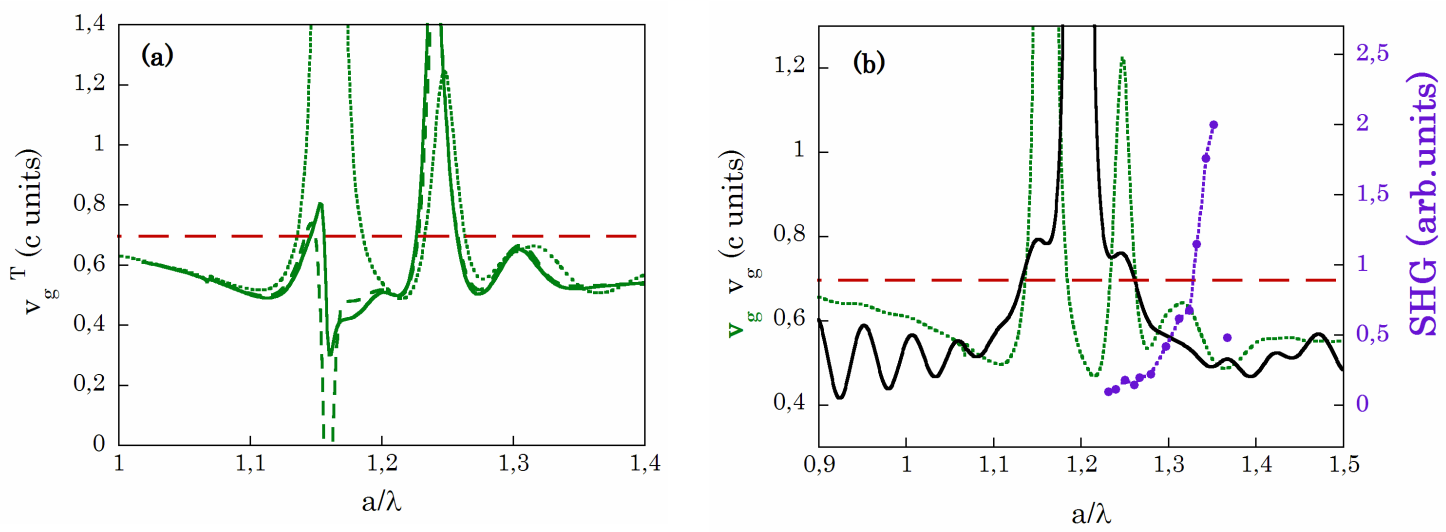

Fig. 1 (a) $v_{g}^{T}$ for 10 (dashed curve), 20 (dashed curve) and 30 (solid curve) [111] planes. (b) $v_{g}^{T}$ and $v_{g}^{R}$ group velocities (black solid curve, green dashed curve), and SHG intensity (violet dotted curve). All plotted as a function of frequency in reduced $\mathrm{a} / \lambda$ units.

In summary, we show that the group velocity slowing-down at the high energy range of 3D photonic nanostructures provides an enhancement mechanism for the nonlinear process.

\section{References}

[1] L. A. Dorado, R. A. Depine, H. Míguez, "Effect of extinction on the high-energy optical response of photonic crystals", Physiscal Review B 75, 241101(2007)

[2] N. Stefanou, V. Karathanos, and A. Modinos, Journal of Physics: Condensed Matter 4, "Scattering of electromagnetic waves by periodic structures" 7389-7400 (1992) N. Stefanou, V. Yannopapas, and A. Modinos, "Heterostructures of photonic crystals: Frequency bands and transmission coefficients" Comput.er Physics Communications 132, 189 (2000) 